\title{
Cognitive and Neurologic Rehabilitation Strategies for Central Nervous System HIV Infection
}

\author{
Terrence Chan $^{1} \cdot$ Monica Marta ${ }^{2,3} \cdot$ Camilla Hawkins $^{1} \cdot$ Simon Rackstraw ${ }^{1,2,3}$
}

Published online: 26 August 2020

(C) The Author(s) 2020

\begin{abstract}
Purpose of Review Cognitive impairment leading to disability is increasingly seen in people living with human immunodeficiency virus (PLWH). Rehabilitation can alleviate the effects of cognitive impairment upon function. The aim of this paper is to discuss the strategies that have been used in cognitive and neurologic rehabilitation in PLWH.

Recent Findings Studies examining pharmacological and non-pharmacological strategies were analysed. Medical management of HIV and co-morbidities should be optimised. Non-pharmacological strategies, including nerve stimulation techniques, exercise-based interventions, and paper and computer-based cognitive rehabilitation, have some evidence supporting their use in PLWH either as stand-alone interventions or as part of a multidisciplinary approach.

Summary Both pharmacological and non-pharmacological rehabilitation strategies have been used with PLWH. More intervention trials are needed to assess cognitive and neurological rehabilitation strategies and further evaluate their potential benefit in PLWH.
\end{abstract}

Keywords Cognitive rehabilitation $\cdot$ Neuro-rehabilitation $\cdot$ HIV-associated neurocognitive disorders $\cdot$ HIV

\section{Introduction}

HIV-associated neurocognitive disorder (HAND) was characterised by severe cognitive dysfunction leading to dementia and death in the pre-antiretroviral era [1]. The introduction of combination antiretroviral therapy (cART) decreased the incidence of the most severe HAND cases [2-4]. It also transformed the management of HIV infection from the symptomatic control of a life-limiting disease to the care of a chronic condition that is associated with a close to normal lifespan [5].

Whilst the most severe forms of HAND are now rare, milder cognitive impairments persist amongst PLWH $[3,6$,

This article is part of the Topical Collection on Central Nervous System and Cognition

Simon Rackstraw

simon.rackstraw@mildmay.org

1 Mildmay Hospital, 19 Tabernacle Gardens, London E2 7DZ, UK

2 Grahame Hayton Unit, I\&I and Neurology Department, Barts Health NHS Trust, London, UK

3 Neurosciences, Blizard Institute, Queen Mary University of London, London, UK
7]. Recent study cohorts suggest that cART does not alter the prevalence of cognitive impairment in PLWH $[6,8,9]$. Furthermore, new HIV-related neurological complications have become apparent with the widespread use of cART. Encephalopathy due to HIV discordance between plasma and cerebrospinal fluid (CSF) [10-12] and CD8 encephalitis [13] is rare but recognisable central nervous system (CNS) presentations seen in PLWH on cART. It has also emerged that some antiretrovirals, such as efavirenz, have direct CNS toxicity $[14,15]$. HIV infection is also associated with a higher risk of cerebro- and cardiovascular disease, either by lifestyle, direct viral or antiretroviral effects [16] (e.g. hyperlipidaemia caused by protease inhibitor-based therapy). Cerebrovascular disease is associated with an increased prevalence of neurological disability and cognitive difficulties. As the life expectancy for PLWH increases due to improved medical management, it has become apparent that some PLWH with neurological and cognitive impairments may survive for many years following acute or ongoing neurological insults.

Rehabilitation strategies are needed to improve quality of life, day to day functional performance and to mitigate further cognitive impairment. Neurological rehabilitation can be defined as a process that aims to optimise health, social functioning and sense of well-being through restorative and 
compensatory strategies [17]. This definition highlights that rehabilitation should be a personalised intervention. It is not a process restricted to people who may recover, partially or completely, but applies to all.

The London Taxi driver study [18•] showed that neuronal volumes can be influenced by tasks, demonstrating the concept of neuroplasticity following interventions. Neurological rehabilitation strategies, particularly those involving multidisciplinary teams (MDT), have been traditionally used in cohorts of people with more static brain injuries, such as traumatic brain injury, stroke or post-hypoxic brain injury [19] but also increasingly in chronic diseases such as multiple sclerosis [20]. These teams include physicians and psychiatrists, rehabilitation nurses, physiotherapists, speech and language therapists, occupational therapists and psychologists.

In this review, we discuss the strategies that have been used in cognitive and neurologic rehabilitation in PLWH.

\section{Methods}

We reviewed outcomes data in our neuro-rehabilitation unit for PLWH.

We used PubMed, Embase, CINAHL, and the Psychology and Behavioural Sciences databases to search for the terms 'HIV', 'cognitive', and 'neurological' and 'rehabilitation'. We reviewed 892 abstracts and selected studies in English that had a clear rehabilitation intervention and described the outcomes in PLWH.

\section{Results}

Whilst many publications looked at cognitive and neurological disability and rehabilitation techniques, the majority did not describe the analysis of an intervention: most of these studies were not randomised, had only small numbers of PLWH and were pilot studies.

We have highlighted studies that demonstrate particular rehabilitation strategies that best illustrate the changes experienced by PLWH following the intervention.

\section{Multidisciplinary Therapy Team Strategies-the Example of Mildmay Hospital}

The Mildmay hospital is an inpatient and day therapy unit in London which is dedicated to the rehabilitation of people with neurological disability caused by or associated with HIV infection. With the use of cART, it evolved from a palliative care unit for people dying with HIV infection to a rehabilitation unit. In the pre-cART era, the brain impairment unit gained experience of delivering neurological and cognitive assessment and care to PLWH admitted for long-term care. Those admitted were very immunocompromised and had neuro-behavioural problems, with severe cognitive impairment secondary to HIV-related neurological disorders (e.g. HIV encephalopathy), opportunistic infections (e.g. cerebral toxoplasmosis or progressive multifocal leukoencephalopathy) or brain tumours. A combination of rehabilitation techniques and cART led to patients with HIV-related brain pathology being discharged with better function rather than dying within the unit [21•].

Currently, PLWH admitted to Mildmay Hospital have been diagnosed with HAND or with cognitive and physical impairments due to neurological opportunistic infections or other HIV-related brain pathology. They tend to have been diagnosed late with HIV infection or have had poor adherence to cART. PLWH are assessed by an MDT that includes an HIV physician, psychiatrist, physiotherapist, occupational therapist, dietitian, social worker, speech and language therapist and psychologist, who design a personalised programme of rehabilitation to meet individual needs and goals.

We measure change during rehabilitation with the Functional Independence Measurement (FIM) and Functional Assessment Measure (FAM) scales adapted for use in the United Kingdom (UK) [22, 23]. These are the principal outcome measure for the UK national database for specialist rehabilitation in patients with complex disabilities [24] (the UK Rehabilitation Outcomes Collaborative (UKROC)). The FAM was developed in the early 1990s for people with traumatic brain injury and includes 12 items that focus on cognitive and psychosocial function [23]. It is used in combination with the 18-item FIM at admission and discharge. The outcomes can be viewed in the form of a 'splat-plot' that change from admission to discharge by the differential area covered, with higher scores indicating better function. An example of the outcomes can be seen in Fig. 1 and Table 1.

An advantage of this multidisciplinary approach to rehabilitation and its evaluation is that it focuses on measuring functional, more ecological outcomes, rather than neurocognitive test scores, and may have more validity to people's daily lives.

\section{‘Medical Maximisation' Strategies with CART}

Although it is now widely accepted that all PLWH regardless of stage of disease should be commenced and maintained on cART [25], this has not always been the case. Due to the toxicities of older regimes, cART was mostly used to treat patients with symptomatic immunosuppression.

Many PLWH benefited cognitively when started on highly active cART, with a global improvement in cognitive domains affected by the disease [8, 26, 27]. On the other hand, there were occasional examples of cohorts that improved cognitively by stopping cART, suggesting a toxicity effect of some medications [28]. Based on this evidence, treatment guidelines suggested that all patients with HIV-related cognitive impairment should be placed on 
Fig. 1 Splat-plot of FIM + FAM domain admission and discharge scores in 2019. FIM, functional independence measure; FAM, functional assessment measure
FIM+FAM splat (Site C126) - 2019/20

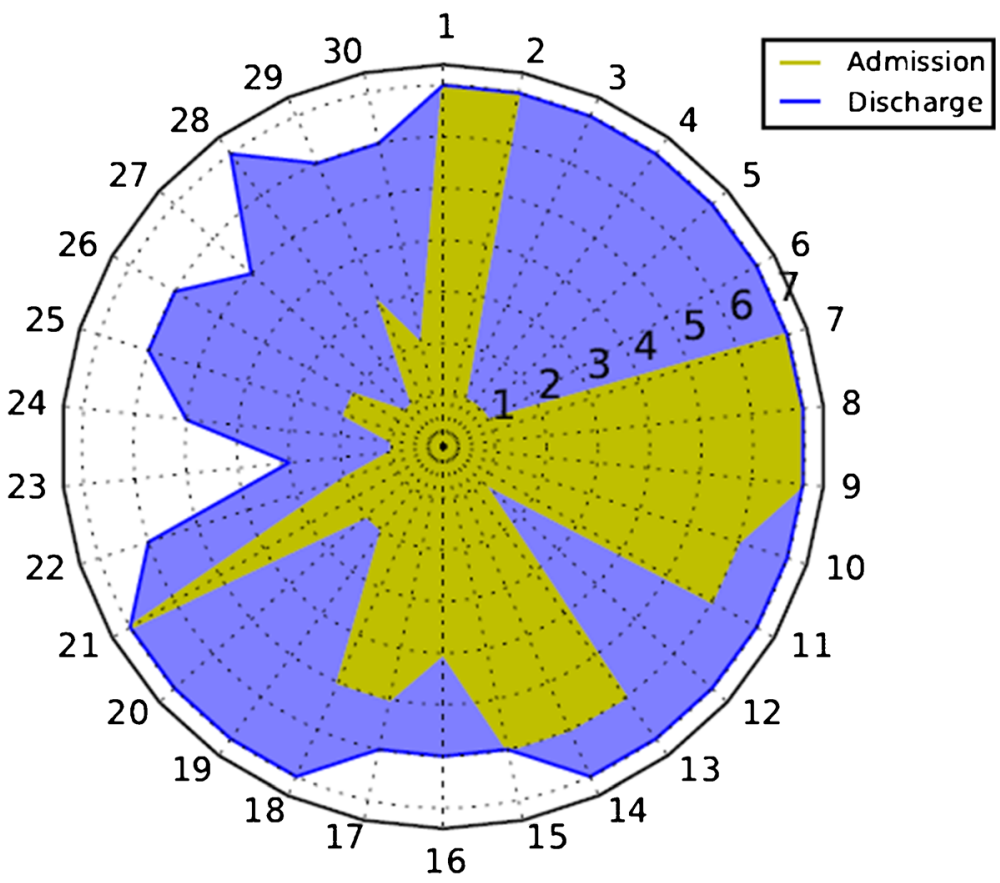

Table 1 Functional gains achieved with multidisciplinary inpatient rehabilitation

\begin{tabular}{lllll}
\hline & Site C126 & & & \\
\cline { 2 - 4 } & $2017 /$ & $2018 /$ & $2019 /$ & Total \\
& 18 & 19 & 20 & \\
\hline Complexity (serial RCS-E scores) & & & & \\
RCS-E v12 score (mean) & 11.2 & 11.4 & 11.1 & 11.3 \\
\% High/very high assessments & $68.0 \%$ & $64.6 \%$ & $60.9 \%$ & $65.5 \%$ \\
Functional gain (FIM + FAM scores) & & & & \\
\% reported & $61 \%$ & $64 \%$ & $25 \%$ & $60 \%$ \\
Motor score on admission (mean) & 90.7 & 87.9 & 67.3 & 88.7 \\
Motor score on discharge (mean) & 99.2 & 96.7 & 79.0 & 97.5 \\
Motor gain during episode (mean) & 8.5 & 8.9 & 11.7 & 8.8 \\
Cognitive score on admission (mean) & 70.9 & 72.8 & 46.3 & 71.0 \\
Cognitive score on discharge (mean) & 77.3 & 80.7 & 65.3 & 78.5 \\
Cognitive gain during episode (mean) & 6.5 & 7.9 & 19.0 & 7.5 \\
FIM + FAM efficiency (median) & 0.2 & 0.1 & 0.0 & 0.1 \\
FIM + FAM efficiency (pop. mean) & 0.2 & 0.2 & 0.4 & 0.2 \\
Reduction in cost of ongoing care & & & & \\
\% reported (optional) & $34 \%$ & $51 \%$ & $75 \%$ & $44 \%$ \\
Mean NPCNA cost/week on admission & $£ 1216$ & $£ 706$ & $£ 606$ & $£ 894$ \\
Mean NPCNA cost/week on discharge & $£ 548$ & $£ 392$ & $£ 73$ & $£ 413$ \\
Mean saving in cost/week & $£ 668$ & $£ 314$ & $£ 532$ & $£ 481$ \\
NPCNA cost efficiency index (median) & 18.4 & 1.5 & 4.0 & 3.8 \\
NPCNA cost efficiency index (pop.median) & 7.4 & 4.7 & 7.2 & 6.1 \\
\hline
\end{tabular}


cART, even at a time when they were unable to make such a recommendation for all patients [29].

Pharmacodynamics and pharmacokinetics of individual ART medications within the CNS differ when compared with the systemic circulation. This underpinned the hypothesis that some drugs lack efficacy in the CNS due to a decreased drug penetration in the CNS compartment and the concept of an ARV regimen CNS Penetration-Effectiveness (CPE) score (the higher the score, the better the CNS penetration) [30]. A number of studies have used this ranking system to examine whether regimes with higher CPE scores have an impact upon PLWH's cognition but the results have been inconsistent, with both positive and negative results reported [31-34].

Another strategy was the addition of maraviroc to the regimes of patients with cognitive impairment. Maraviroc has an effect on neuro-inflammation and monocyte activity, and was used as an adjunct in virologically suppressed patients, with some success in small pilot studies [35,36]. Other cART optimisation strategies include a switch from regimes that have been associated with poor neurocognitive performance, particularly efavirenz. Neuropsychological symptoms, and in some cases objective neuropsychological dysfunction, have been noted as side effects of using efavirenz in cART regimes [15], and switching from efavirenz-containing regimes has been associated with improved well-being but not always cognitive benefit in formal testing [37].

Recently, a differentially resistant virus in CSF and plasma (HIV compartmentalisation) has been found in PLWH with neurologically symptomatic disease. Although symptomatic HIV CSF escape is rare $[10,11]$, modification of cART according to the genotypes of both plasma and CSF compartments may improve cognitive symptoms amongst these patients.

\section{'Medical Maximisation' Strategies-Management of Co-Morbidities}

There continues to be some debate about the aetiology of cognitive difficulties in HIV infection and the extent to which other medical problems that are affecting PLWH also compound the effects of HAND [3].

Co-infections have been associated with neurocognitive impairments, and therefore require effective management. Hepatitis $\mathrm{C}$ infection is associated with neurocognitive impairments in both mono-infected patients and in those co-infected with HIV infection [38, 39]. There have been some studies demonstrating some neurocognitive benefit in virological clearance of hepatitis $\mathrm{C}$ with both interferon and direct acting agents [40, 41].

Poorly controlled cardiovascular risk factors such as diabetes or hypertension are associated with poorer cognitive outcomes in PLWH [36-39]. Studies in improved management of those diseases have shown mixed results in improving cognition [42].

Many cohort studies show high levels of psychiatric disorders in PLWH. Depression and anxiety can mask or resemble symptoms of cognitive impairment. In addition, severe mental health difficulties may affect performance in neurocognitive tests and the ability of PLWH to perform day to day tasks. There is some evidence that neurocognitive functions can be improved by active treatment of depression with some antidepressants [43].

There has also been interest in the treatment of HAND by the addition of other adjunctive medications to try to ameliorate the cytokine changes associated with HAND [44-47]. The effects in pilot studies have been variable, but usually disappointing, showing very little real cognitive change.

\section{Nerve Stimulation Techniques}

A number of different nerve stimulation techniques have been proposed as adjunctive methods as part of neuro-rehabilitation. Techniques such as binaural beat therapy have been used experimentally to treat anxiety and improve cognitive processing. Similarly, vagal nerve stimulation has been examined as a treatment for both refractory depression and epilepsy: some of the patients treated in these studies had some improvements in attention and memory recall. Neither of these techniques has yet been used in PLWH.

However, transcranial direct current stimulation has been trialled in PLWH. Electrodes are placed cutaneously over parts of the scalp corresponding to neural networks, and are thought to influence change in neuronal activity or neurotransmitter release. This was tested in a driving simulation study, combined with speed of processing cognitive remediation therapy in a randomised 1:1 fashion between two groups of 15 unimpaired PLWH. Results suggested more cautious driving behaviour following active intervention. People in the training group showed fewer lane deviations and a slower average driving speed, in contrast to the control group who showed an increase in driving deviations and average driving speed [48].

A combination of techniques was tested further in a group of 33 older ( $>50$ years) PLWH. The participants underwent neuropsychological testing and training with combination speed of processing cognitive remediation therapy, and were then randomised to receive either real transcranial direct stimulation $(n=17)$ or not $(n=16)$. The neuropsychological testing after the intervention showed small-to-medium effects in the executive and speed of processing measure. Medium-tolarge effects were observed for an executive/attention and oral reading measure. There were small-to-medium and mediumto-large effects for two speed of processing measures in the opposite direction (i.e. the control group showed greater improvements). However, the only statistically significant effect 
was seen on the oral reading measure [49]. It is a technique that needs further studies to examine it further as part of a neuro-rehabilitation strategy.

\section{Exercise-Based Interventions}

Exercise- or physical activity-based interventions were used to both improve cognitive abilities, or to prevent cognitive decline in PLWH. There is already a body of data attesting to the benefits of exercise on cognition in non-HIV populations $[50,51]$.

A small study randomised 11 PLWH who were not cognitively impaired at baseline into either an exercise group $(n=5)$ who completed a 16-week aerobic exercise programme training 3 times per week or a control non-exercise group $(n=6)$. The results showed higher moderate physical activity correlated with higher MOCA scores, but exercise training did not induce significant improvements in cognitive function [52]. It is important to note that adherence to the intervention was only $60 \%$.

Yoga as a strategy for cognitive improvement was tested in 18 adolescent PLWH at a rehabilitation centre in India. It was a single-group study with a pre- and post-intervention assessment of general health, immune parameters, quality of life and cognitive functioning. All the participants were all given $1 \mathrm{~h}$ of yoga daily for 6 months. The results demonstrated an improvement in general health of the participants. However, the cognitive function analysis had mixed results with improved psychomotor performance but worsened executive functioning [53]. This is a strategy which has seen some promising results in the general older population and deserves to be studied further.

\section{Paper-Based Cognitive Rehabilitation}

A study from San Diego used Goal Management Training (GMT) and metacognitive training as a strategy for improving executive function difficulties, seen in people with HAND and in people with substance use. GMT showed efficacy in improving executive function difficulties in daily life. Metacognition or 'thinking about thinking' is based on an active awareness of cognitive functions and understanding of cognitive difficulties, which is also often suboptimal in those with HAND and those who use substances.

Ninety PLWH with a previous or current history of substance use and current executive function difficulties were randomised 1:1:1 to either receive a single session of Goal Management Training or a single session of both Goal Management Training plus Metacognitive Training or to an active control group, with 30 participants in each group [54]. The results suggest that a brief, single session of Goal Management Training (GMT) protocol has some effect on multitasking abilities.
An Italian study is an example of the combination of paperbased and computerised cognitive-based exercises as a cognitive rehabilitation strategy. Thirty-two PLWH (16 with and 16 without HAND) were randomised 1:1 to the intervention (eight different exercises, lasting about $50 \mathrm{~min}$ in duration, which were repeated 36 times over a 4-month period) or as a control. Neurocognitive assessment was performed before, and after the study in all participants, and six months after the completion of the intervention for those in the interventional arm. The intervention was directed at improving abilities within four cognitive domains: attention, visual-verbal memory and learning, executive functioning and working memory, and metacognitive awareness. The intervention group demonstrated improvements in five of eight cognitive domains measured, including learning and memory, abstraction/executive function, verbal fluency, attention, and functional tasks, whereas those in the control group showed a deterioration in those domains. When re-measured after 6 months, the intervention group had maintained improvements in abstraction/executive functioning, attention/ working memory and functional tasks [55••]. This demonstrates that patients can participate in a cognitive rehabilitation protocol, make cognitive gains and maintain them, including in functional tasks 6 months after completion of the programme.

\section{Computer-Based Strategies}

Computerised neuropsychological assessments have become increasingly common in recent years. There has been particular interest in brief screens and their use in intervention studies. This is because of the speed of execution and decreased cost compared with traditional full neuropsychological assessments. They have been validated in several disease areas including in PLWH with various degrees of cognitive impairment. There has also been some interest in using computerised cognitive rehabilitation programmes, as these potentially have the advantage of being able to be performed in the community without supervision. A couple of studies have shown some potential advantages and disadvantages of the approach.

In a partially randomised study of 30 PLWH and 30 HIVnegative adults, those assigned to the intervention group were asked to use SmartBrain (SmartBrain Technologies, 2013) from their home computer for 24 weeks, initially for $10 \mathrm{~min}$ and increasing weekly to a maximum of $30 \mathrm{~min}$ [56]. SmartBrain contains 14 game-like modules in the domains of memory, attention, knowledge and executive functions. Participants were tested with an extensive battery of neurocognitive assessments before and after the intervention. Primary results from this study revealed no significant effects of SmartBrain on global cognitive outcomes, irrespective of HIV serostatus. However, adherence to the intervention was 
poor with only $54 \%$ of participants able to use the programme more than once. A post hoc analysis showed that the most benefit was gained by those who undertook the intervention most frequently.

In Uganda, 1596 to 12-year-old HIV positive children were randomised 1:1:1 to a group either receiving a computerised rehabilitation programme using computer games that was progressively more difficult, to a limited programme that generated games of random degrees of difficulty, or no computerised intervention, over a 2 -month period [57••]. The computerised rehabilitation package was called Captain's Log (BrainTrain Corporation) programmed with games to improve working memory, attention and visual-spatial analysis. Planning and knowledge domains had significantly greater gains at follow-up in the full intervention group when compared with controls. Both intervention arms had significant improvements on learning tests compared with controls. This demonstrates that this could be a strategy for use in resource-poor country situations.

\section{Conclusion}

Cognitive impairment is still prevalent in PLWH in the cART era, and can lead to disability affecting activities of daily living. Both pharmacological and non-pharmacological rehabilitation strategies are currently being increasingly used to minimise the effect of such impairments. Early studies suggest promise in remediating some of the cognitive impairment caused by HIV. More intervention trials are needed to assess cognitive and neurological rehabilitation strategies and further evaluate their benefit in PLWH.

\section{Compliance with Ethical Standards}

Conflict of Interest The authors declare that they have no conflicts of interest.

Human and Animal Rights and Informed Consent This article does not contain any studies with human or animal subjects performed by any of the authors.

Open Access This article is licensed under a Creative Commons Attribution 4.0 International License, which permits use, sharing, adaptation, distribution and reproduction in any medium or format, as long as you give appropriate credit to the original author(s) and the source, provide a link to the Creative Commons licence, and indicate if changes were made. The images or other third party material in this article are included in the article's Creative Commons licence, unless indicated otherwise in a credit line to the material. If material is not included in the article's Creative Commons licence and your intended use is not permitted by statutory regulation or exceeds the permitted use, you will need to obtain permission directly from the copyright holder. To view a copy of this licence, visit http://creativecommons.org/licenses/by/4.0/.

\section{References}

Papers of particular interest, published recently, have been highlighted as:

- Of importance

- Of major importance

1. Navia BA, Jordan BD, Price RW. The AIDS dementia complex: I Clinical features. Ann Neurol. 1986 Jun;19(6):517-24.

2. Sacktor N, McDermott MP, Marder K, Schifitto G, Selnes OA, McArthur JC, et al. HIV-associated cognitive impairment before and after the advent of combination therapy. Journal of NeuroVirology. 2002;8(2):136-42.

3. Heaton RK, Clifford DB, Franklin DR, Woods SP, Ake C, Vaida F, et al. HIV-associated neurocognitive disorders persist in the era of potent antiretroviral therapy. Neurology. 2010;75(23):2087-96.

4. The UK, Collaborative HIV. Cohort (CHIC) Study Steering Committee. HIV-associated central nervous system diseases in the recent combination antiretroviral therapy era. Eur J Neurol. 2011;18(3):527-34.

5. May MT, Gompels M, Delpech V, Porter K, Orkin C, Kegg S, et al. Impact on life expectancy of HIV-1 positive individuals of CD4+ cell count and viral load response to antiretroviral therapy. AIDS. 2014;28(8):1193-202.

6. Heaton RK, Franklin DR, Ellis RJ, McCutchan JA, Letendre SL, LeBlanc S, et al. HIV-associated neurocognitive disorders before and during the era of combination antiretroviral therapy: differences in rates, nature, and predictors. Journal of NeuroVirology. 2011;17(1):3-16.

7. Bonnet F, Amieva H, Marquant F, Bernard C, Bruyand M, Dauchy F-A, et al. Cognitive disorders in HIV-infected patients: are they HIV-related? AIDS. 2013;27(3):391-400.

8. Tozzi V, Balestra P, Bellagamba R, Corpolongo A, Salvatori MF, Visco-Comandini U, et al. Persistence of neuropsychologic deficits despite long-term highly active antiretroviral therapy in patients with HIV-related neurocognitive impairment: prevalence and risk factors. JAIDS Journal of Acquired Immune Deficiency Syndromes. 2007;45(2):174-82.

9. Simioni S, Cavassini M, Annoni J-M, Rimbault Abraham A, Bourquin I, Schiffer V, et al. Cognitive dysfunction in HIV patients despite long-standing suppression of viremia. AIDS. 2010;24(9): 1243-50.

10. Canestri A, Lescure F-X, Jaureguiberry S, Moulignier A, Amiel C, Marcelin A, et al. Discordance between cerebral spinal fluid and plasma HIV replication in patients with neurological symptoms who are receiving suppressive antiretroviral therapy. Clin Infect Dis. 2010;50(5):773-8.

11. Peluso MJ, Ferretti F, Peterson J, Lee E, Fuchs D, Boschini A, et al. Cerebrospinal fluid HIV escape associated with progressive neurologic dysfunction in patients on antiretroviral therapy with well controlled plasma viral load. AIDS. 2012;26(14):1765-74.

12. Mukerji SS, Misra V, Lorenz DR, Uno H, Morgello S, Franklin D, et al. Impact of antiretroviral regimens on cerebrospinal fluid viral escape in a prospective multicohort study of antiretroviral therapyexperienced human immunodeficiency virus-1-infected adults in the United States. Clin Infect Dis. 2018;67(8):1182-90.

13. Lescure F-X, Moulignier A, Savatovsky J, Amiel C, Carcelain G, Molina J-M, et al. CD8 encephalitis in HIV-infected patients receiving cART: a treatable entity. Clin Infect Dis. 2013;57(1):101-8.

14. Robertson K, Liner J, Meeker RB. Antiretroviral neurotoxicity. Journal of NeuroVirology. 2012;18(5):388-99.

15. Ciccarelli N, Fabbiani M, Di Giambenedetto S, Fanti I, Baldonero E, Bracciale L, et al. Efavirenz associated with cognitive disorders 
in otherwise asymptomatic HIV-infected patients. Neurology. 2011;76(16):1403-9.

16. Gutierrez J, Albuquerque ALA, Falzon L. HIV infection as vascular risk: a systematic review of the literature and meta-analysis. PLoS One. 2017;12(5):e0176686.

17. Krucoff MO, Rahimpour S, Slutzky MW, Edgerton VR, Turner DA. Enhancing nervous system recovery through neurobiologics, neural interface training, and neurorehabilitation. Front Neurosci. 2016;10:584.

18. Maguire EA, Woollett K, Spiers HJ. London taxi drivers and bus drivers: a structural MRI and neuropsychological analysis. Hippocampus. 2006;16(12):1091-101 Important paper that demonstrates the concepts of neuroplasticity in action.

19. Cappa SF, Benke T, Clarke S, Rossi B, Stemmer B, van Heugten $\mathrm{CM}$, et al. EFNS guidelines on cognitive rehabilitation: report of an EFNS task force. Eur J Neurol. 2005 Sep;12(9):665-80.

20. Amatya B, Khan F, Galea M. Rehabilitation for people with multiple sclerosis: an overview of Cochrane Reviews. Cochrane Database Syst Rev. 2019;1:CD012732.

21. Stephenson J, Woods S, Scott B, Meadway J. HIV-related brain impairment from palliative care to rehabilitation. Int J Palliat Nurs. 2000;6(1):6-11 First paper exploring neuro-rehabilitation in patients with HIV related cognitive impairment.

22. Turner-Stokes L, Nyein K, Turner-Stokes T, Gatehouse C. The UK FIM+FAM: development and evaluation. Clin Rehabil. 1999;13(4):277-87.

23. Law J, Fielding B, Jackson D, Turner-Stokes L. The UK FIM+ FAM extended activities of daily living module: evaluation of scoring accuracy and reliability. Disabil Rehabil. 2009;31(10):825-30.

24. Turner-Stokes L, Williams H, Sephton K, Rose H, Harris S, Thu A. Engaging the hearts and minds of clinicians in outcome measurement - the UK rehabilitation outcomes collaborative approach. Disabil Rehabil. 2012;34(22):1871-9.

25. The INSIGHT START Study Group. Initiation of antiretroviral therapy in early asymptomatic HIV infection. N Engl J Med. 2015;373(9):795-807.

26. Ferrando S, van Gorp W, McElhiney M, Goggin K, Sewell M, Rabkin J. Highly active antiretroviral treatment in HIV infection: benefits for neuropsychological function. AIDS. 1998;12(8):F6570.

27. Cysique LA, Vaida F, Letendre S, Gibson S, Cherner M, Woods $\mathrm{SP}$, et al. Dynamics of cognitive change in impaired HIV-positive patients initiating antiretroviral therapy. Neurology. 2009;73(5): 342-8.

28. Robertson KR, Su Z, Margolis DM, Krambrink A, Havlir DV, Evans S, et al. Neurocognitive effects of treatment interruption in stable HIV-positive patients in an observational cohort. Neurology. 2010;74(16):1260-6.

29. Williams I, Churchill D, Anderson J, Boffito M, Bower M, Cairns $\mathrm{G}$, et al. British HIV Association guidelines for the treatment of HIV-1-positive adults with antiretroviral therapy 2012 (Updated November 2013. All changed text is cast in yellow highlight). HIV Medicine. 2014;15(S1):1-6.

30. Letendre S, Marquie-Beck J, Capparelli E, Best B, Clifford D, Collier AC, et al. Validation of the CNS penetration-effectiveness rank for quantifying antiretroviral penetration into the central nervous system. Arch Neurol. 2008;65(1):65-70.

31. Marra CM, Zhao Y, Clifford DB, Letendre S, Evans S, Henry K, et al. Impact of combination antiretroviral therapy on cerebrospinal fluid HIV RNA and neurocognitive performance. AIDS. 2009;23(11):1359-66.

32. Fabbiani M, Grima P, Milanini B, Mondi A, Baldonero E, Ciccarelli N, et al. Antiretroviral neuropenetration scores better correlate with cognitive performance of HIV-infected patients after accounting for drug susceptibility. Antivir Ther. 2014;20(4):441-7.
33. Baker LM, Paul RH, Heaps-Woodruff JM, Chang JY, Ortega M, Margolin Z, et al. The effect of central nervous system penetration effectiveness of highly active antiretroviral therapy on neuropsychological performance and neuroimaging in HIV infected individuals. J NeuroImmune Pharmacol. 2015 Sep 1;10(3):487-92.

34. Santos GMA, Locatelli I, Métral M, Calmy A, Lecompte TD, Nadin I, et al. cross-sectional and cumulative longitudinal central nervous system penetration effectiveness scores are not associated with neurocognitive impairment in a well treated aging human immunodeficiency virus-positive population in Switzerland. Open Forum Infect Dis [Internet]. 20191 [cited 2019 Dec 3];6(7). Available from: https://academic.oup.com/ofid/article/6/7/ofz277/ 5529443. Accessed 13 Nov 2019.

35. Ndhlovu LC, Umaki T, Chew GM, Chow DC, Agsalda M, Kallianpur KJ, et al. Treatment intensification with maraviroc (CCR5 antagonist) leads to declines in CD16-expressing monocytes in cART-suppressed chronic HIV-infected subjects and is associated with improvements in neurocognitive test performance: implications for HIV-associated neurocognitive disease (HAND). Journal of NeuroVirology. 2014;20(6):571-82.

36. Gates TM, Cysique LA, Siefried KJ, Chaganti J, Moffat KJ, Brew BJ. Maraviroc-intensified combined antiretroviral therapy improves cognition in virally suppressed HIV-associated neurocognitive disorder. AIDS. 2016 Feb 20;30(4):591-600.

37. Hakkers CS, Arends JE, van den Berk GE, Ensing MHM, Hooijenga I, Vink M, et al. Objective and subjective improvement of cognition after discontinuing efavirenz in asymptomatic patients: a randomized controlled trial. J Acquir Immune Defic Syndr. 2019;80(1):e14-22.

38. Forton DM, Thomas HC, Murphy CA, Allsop JM, Foster GR, Main J, et al. Hepatitis $\mathrm{C}$ and cognitive impairment in a cohort of patients with mild liver disease. Hepatology. 2002;35(2):433-9.

39. Hinkin CH, Castellon SA, Levine AJ, Barclay TR, Singer EJ. Neurocognition in individuals co-infected with HIV and hepatitis C. J Addict Dis. 2008;27(2):11-7.

40. Thein HH, Maruff P, Krahn MD, Kaldor JM, Koorey DJ, Brew BJ, et al. Improved cognitive function as a consequence of hepatitis $\mathrm{C}$ virus treatment. HIV Medicine. 2007;8(8):520-8.

41. Kleefeld F, Heller S, Ingiliz P, Jessen H, Petersen A, Kopp U, et al. Interferon-free therapy in hepatitis $\mathrm{C}$ virus $(\mathrm{HCV})$ monoinfected and $\mathrm{HCV} / \mathrm{HIV}$ coinfected patients: effect on cognitive function, fatigue, and mental health. Journal of NeuroVirology. 2018;24(5): 557-69.

42. Foley J, Ettenhofer M, Wright MJ, Siddiqi I, Choi M, Thames AD, et al. Neurocognitive functioning in HIV-1 infection: effects of cerebrovascular risk factors and age. Clin Neuropsychol. 2010;24(2):265-85.

43. Letendre SL, Marquie-Beck J, Ellis RJ, Woods SP, Best B, Clifford $\mathrm{DB}$, et al. The role of cohort studies in drug development: clinical evidence of antiviral activity of serotonin reuptake inhibitors and HMG-CoA reductase inhibitors in the central nervous system. Jrnl Neuroimmune Pharm. 2007;2(1):120-7.

44. Simioni S, Cavassini M, Annoni J-M, Métral M, Iglesias K, Rimbault Abraham A, et al. Rivastigmine for HIV-associated neurocognitive disorders: a randomized crossover pilot study. Neurology. 2013;80(6):553-60.

45. Letendre SL, Woods SP, Ellis RJ, Atkinson JH, Masliah E, van den Brande G, et al. Lithium improves HIV-associated neurocognitive impairment. AIDS. 2006;20(14):1885-8.

46. Probasco JC, Spudich SS, Critchfield J, Lee E, Lollo N, Deeks SG, et al. Failure of atorvastatin to modulate CSF HIV-1 infection. Neurology. 2008;71(7):521-4.

47. Sacktor N, Miyahara S, Deng L, Evans S, Schifitto G, Cohen BA, et al. Minocycline treatment for HIV-associated cognitive impairment. Neurology. 2011;77(12):1135-42. 
48. Pope CN, Stavrinos D, Vance DE, Woods AJ, Bell TR, Ball KK, et al. A pilot investigation on the effects of combination transcranial direct current stimulation and speed of processing cognitive remediation therapy on simulated driving behavior in older adults with HIV. Transport Res F: Traffic Psychol Behav. 2018 Oct;58:106173.

49. Fazeli PL, Woods AJ, Pope CN, Vance DE, Ball KK. Effect of transcranial direct current stimulation combined with cognitive training on cognitive functioning in older adults with HIV: a pilot study. Applied Neuropsychology: Adult. 2019;26(1):36-47.

50. Hötting K, Röder B. Beneficial effects of physical exercise on neuroplasticity and cognition. Neurosci Biobehav Rev. 2013;37(9, Part B):2243-57.

51. Hishikawa N, Takahashi Y, Fukui Y, Tokuchi R, Furusawa J, Takemoto M, et al. Yoga-plus exercise mix promotes cognitive, affective, and physical functions in elderly people. Neurol Res. 2019;41(11):1001-7.

52. McDermott A, Zaporojan L, McNamara P, Doherty CP, Redmond $\mathrm{J}$, Forde $\mathrm{C}$, et al. The effects of a 16 -week aerobic exercise programme on cognitive function in people living with HIV. AIDS Care. 2017;29(6):667-74.

53. Hari Chandra B, Ramesh MN, Nagendra HR. Effect of yoga on immune parameters, cognitive functions, and quality of life among HIV-positive children/adolescents: a pilot study. Int J Yoga. 2019;12(2):132-8.

54. Casaletto KB, Moore DJ, Woods SP, Umlauf A, Scott JC, Heaton RK. Abbreviated goal management training shows preliminary evidence as a neurorehabilitation tool for HIV-associated neurocognitive disorders among substance users. Clin Neuropsychol. 2016;30(1):107-30.

55.• Livelli A, Orofino GC, Calcagno A, Farenga M, Penoncelli D, Guastavigna M, et al. Evaluation of a cognitive rehabilitation protocol in HIV patients with associated neurocognitive disorders: efficacy and stability over time. Front Behav Neurosci [Internet]. 2015 Nov 16 [cited 2019 Dec 3];9. Available from: http://journal. frontiersin.org/Article/10.3389/fnbeh.2015.00306/abstract. Accessed 13 Nov 2019. Important study showing the impact of a cognitive rehabilitation protocol in in patients with HAND.

56. Becker JT, Dew MA, Aizenstein HJ, Lopez OL, Morrow L, Saxton $J$, et al. A pilot study of the effects of internet-based cognitive stimulation on neuropsychological function in HIV disease. Disabil Rehabil. 2012 Oct 1;34(21):1848-52.

57.• Boivin MJ, Nakasujja N, Sikorskii A, Opoka RO, Giordani B. A randomized controlled trial to evaluate if computerized cognitive rehabilitation improves neurocognition in Ugandan children with HIV. AIDS Res Hum Retrovir. 2016;32(8):743-55 Important study demonstrating the ability to execute and the effect of a computer based neurocognitive rehabilitation intervention in a resource poor setting.

Publisher's Note Springer Nature remains neutral with regard to jurisdictional claims in published maps and institutional affiliations. 